\title{
Association of Hospitalised Infection With Socioeconomic Status in Patients With Rheumatoid Arthritis Receiving Biologics or Tofacitinib: A Population-Based Cohort Study
}

OPEN ACCESS

Edited by:

Kayo Masuko,

Sanno Medical Center, Japan

Reviewed by:

Satoshi Kubo,

University of Occupational and Environmental Health Japan, Japan

Ettore Silvagni,

University of Ferrara, Italy

*Correspondence:

Chen-Yu Wang

chestmen@gmail.com

Wen-Cheng Chao

cwc081@hotmail.com

Specialty section:

This article was submitted to

Rheumatology,

a section of the journal

Frontiers in Medicine

Received: 16 April 2021

Accepted: 07 June 2021

Published: 12 July 2021

Citation:

Chen H-H, Lin C-H, Wang C-Y and

Chao W-C (2021) Association of

Hospitalised Infection With

Socioeconomic Status in Patients

With Rheumatoid Arthritis Receiving

Biologics or Tofacitinib: A

Population-Based Cohort Study.

Front. Med. 8:696167.

doi: 10.3389/fmed.2021.696167

\author{
Hsin-Hua Chen ${ }^{1,2,3,4,5,6,7}$, Ching-Heng Lin ${ }^{1,4,6,8,9}$, Chen-Yu Wang ${ }^{10,11 *}$ and \\ Wen-Cheng Chao ${ }^{7,10,12,13 *}$
}

${ }^{1}$ Department of Medical Research, Taichung Veterans General Hospital, Taichung, Taiwan, ${ }^{2}$ Division of Allergy, Immunology and Rheumatology, Department of Internal Medicine, Taichung Veterans General Hospital, Taichung, Taiwan, ${ }^{3}$ Institute of Biomedical Science and Rong Hsing Research Centre for Translational Medicine, Chung Hsing University, Taichung, Taiwan, ${ }^{4}$ Department of Industrial Engineering and Enterprise Information, Tunghai University, Taichung, Taiwan, ${ }^{5}$ School of Medicine, China Medical University, Taichung, Taiwan, ${ }^{6}$ Institute of Public Health and Community Medicine Research Center, National Yang-Ming University, Taipei, Taiwan, ${ }^{7}$ Big Data Center, Chung Hsing University, Taichung, Taiwan, ${ }^{8}$ Department of Healthcare Management, National Taipei University of Nursing and Health Sciences, Taipei, Taiwan, ${ }^{9}$ Department of Public Health, College of Medicine, Fu Jen Catholic University, New Taipei City, Taiwan, ${ }^{10}$ Department of Critical Care Medicine, Taichung Veterans General Hospital, Taichung, Taiwan, ${ }^{11}$ Department of Nursing, Hung Kuang University, Taichung, Taiwan, ${ }^{12}$ Department of Computer Science, Tunghai University, Taichung, Taiwan, ${ }^{13}$ Department of Automatic Control Engineering, Feng Chia University, Taichung, Taiwan

Objectives: Use of biologics or targeted synthetic disease-modifying anti-rheumatic drugs (b/tsDMARDs) is associated with infection in patients with rheumatoid arthritis (RA). Socioeconomic status is substantial in infectious diseases; however, the impact of socioeconomic status on risk for infection in patients with RA receiving b/tsDMARD remains unclear.

Methods: We used the 2003-2017 Taiwanese National Health Insurance Research Database to identify patients with RA receiving b/tsDMARDs. A Cox regression analysis was used to estimate the associations of covariates with the risk of hospitalised infection shown as hazard ratios (HRs) with 95\% confidence interval (Cls).

Results: We identified 7,647 RA patients who started their first bDMARD/tsDMARD treatment. Log-rank analyses demonstrated the association between age $(p<0.001)$, urbanisation $(p=0.001)$, the insured amount $(p=0.021)$, and the hospitalisation. Cox proportional regression analyses showed that age was independently associated with hospitalised infection in a dose-response manner, whereas a high-income category had an inverse association (HR 0.48, 95\% Cl 0.23-0.96). Hospitalisation for infection within 5 years was a strong risk factor (HR 5.63, 95\% Cl 1.91-16.62), and living in a rural area tended to be a risk factor ( $\mathrm{HR} 1.76,95 \% \mathrm{Cl}$ 0.98-3.14) for incident hospitalised infection.

Conclusions: This study showed the crucial impacts of age, socioeconomic status, and history of infection on hospitalised infection in patients with RA receiving b/tsDMARDs. These findings highlight the largely ignored role of socioeconomic status in risk stratification among patients receiving b/tsDMARDs for RA.

Keywords: rheumatoid arthritis, biologics, infection, risk factors, socioeconomic status 


\section{BACKGROUND}

Rheumatoid arthritis (RA) affects $0.5-1 \%$ of the global population and ranks as the 42nd highest contributor to the global disability with an enormous economic impact due to devastating arthritis and disability (1-3). A remarkable advance in the management for RA has been achieved after the development of biological disease-modifying anti-rheumatic drugs (bDMARDs), so-called biologics, and targeted synthetic DMARD (tsDMARD) in the past two decades $(4,5)$. The efficacy of b/tsDMARDs in the management of RA is well-established; however, an increased risk for infection remains a major concern (6-11). Accumulating evidence has shown the essential impact of socioeconomic status on the risk for infection in general populations $(12,13)$, but few studies have addressed the impact of socioeconomic status on risk for infection in patients with $\mathrm{RA}$ receiving $\mathrm{b} / \mathrm{tsDMARDs}(10)$. In the present populationbased study in Taiwan, we enrolled biologics-naïve patients with RA and received b/tsDMARDs between 2003 and 2017 to address risk factors, including income and urbanisation level, for infection requiring hospitalisation.

\section{METHODS}

\section{Ethics Approval}

The Institutional Review Board (IRB) of Taichung Veterans General Hospital (IRB TCVGH number: CE19038A) approved this study. Informed consent was waived, given that all of the personal information had been anonymised before data analyses.

\section{Study Design}

The study is a retrospective cohort study.

\section{Data Source}

This study utilised the 2003-2017 claim data from the National Health Insurance Research Database (NHIRD). Taiwan implemented an obligatory National Health Insurance (NHI) program in 1995 . The NHI program has since then covered over $99.6 \%$ of the population in Taiwan in 2017 (14). The data of the NHIRD contains comprehensive claims data regarding the information on registration, demographic characteristics, residence, prescriptions, diagnosis, examinations, procedures, medical expenditures, outpatient services, and inpatient services. The Bureau of NHI (BNHI) has improved the accuracy of claims data in the NHIRD by checking original medical records regularly (15). The BNHI established a registry for catastrophic illness patients (RCIP) for those who have severe or major diseases, such as RA and cancers, and patients with a certificate for the RCIP were exempt from copayment. Notably, a certificate for RCIP was only issued after validation by at least two qualified specialists through a comprehensive review of original medical charts. We utilised multiple NHIRD datasets to conduct this study, including 2003-2017 outpatient and inpatient claims files and RCIP enrolment files.

\section{Study Subjects}

We identified patients with biologics-naïve patients with RA newly treated with b/tsDMARD during 2003-2017 as study subjects. We defined mutually exclusive categories based on the initiated treatment: (1) tumour necrosis factor inhibitors (TNFis) (etanercept, adalimumab, and golimumab), (2) non-TNFi bDMARDs (abatacept and tocilizumab), and (3) tsDMARD (tofacitinib). The index date was the first date of bDMARD/tsDMARD prescription.

\section{Outcome}

The outcome was the time from the index date to the time of the first inpatient visit with a diagnosis of infection (Supplementary Table 1). We defined the censored date as 90 days after the last date of bDMARD/tsDMARD prescription, switching to another b/tsDMARD, December 31, 2017 (the last date of the data used in this study), or the time of withdrawal from the NHI for any reason, such as leaving or death, whichever came first. The follow-up duration was from the index date to the date of hospitalised infection occurrence or from the index date to the censored date. We calculated the incidence of hospitalised infection by dividing the number of incident hospitalised infection cases by the sum of the followup durations.

\section{Covariates}

Potential predictors for the risk of hospitalised infection included baseline socioeconomic status, sex, age at the index date, comorbidities, medication use within 6 months before the index date, and medication use during the follow-up period. The studied medications included non-steroidal anti-inflammatory drugs (NSAIDs), glucocorticosteroids, methotrexate, sulfasalazine, leflunomide, hydroxychloroquine, and immunosuppressants (i.e., cyclosporine, azathioprine). The presence of comorbidities was defined as having $\geq 3$ outpatient visits or $\geq 1$ hospitalisation with the corresponding International Classification of Diseases-9-Clinical Modification (ICD-9-CM) code or ICD-10-CM code within 1 year before the index date. We used the urbanisation level and insured amount to explore the impact of socioeconomic status. The urbanisation level of the patients' residence was categorised into two levels based on population density (people $/ \mathrm{km}^{2}$ ), population ratio of elderly subjects aged $\geq 65$ years, population ratio of subjects with educational levels of college or above, population ratio of agricultural workers, and number of physicians/100,000 subjects (16). The payroll-related insured amount was categorised into an ordinal variable with three levels in accordance with the payroll bracket categories of NHI program in Taiwan.

\section{Statistical Analysis}

We presented continuous variables as a mean \pm standard deviation and categorical variables as a percentage of patients. We examined the differences in continuous variables by Student's $t$ test and categorical variables by Pearson's $\chi^{2}$ test. The primary dependent variable in this study was hospitalised infection incidence, and the incidence rates (per 100,000 person-year) 
TABLE 1 | Characteristics of enrolled subjects with RA receiving b/tsDMARDs.

\begin{tabular}{|c|c|c|c|c|c|c|c|}
\hline & $\begin{array}{c}\text { All } \\
7,647\end{array}$ & $\begin{array}{c}\text { Etanercept } \\
2,862\end{array}$ & $\begin{array}{c}\text { Adalimumab } \\
2,289\end{array}$ & $\begin{array}{c}\text { Golimumab } \\
912\end{array}$ & $\begin{array}{l}\text { Tocilizumab } \\
502\end{array}$ & $\begin{array}{c}\text { Abatacept } \\
563\end{array}$ & $\begin{array}{c}\text { Tofacitinib } \\
519\end{array}$ \\
\hline \multicolumn{8}{|l|}{ Demographic data } \\
\hline Age at initiating b/tsDMARDs, years & $53.9 \pm 12.8$ & $53.3 \pm 12.7$ & $53.6 \pm 12.8$ & $54.0 \pm 12.6$ & $54.6 \pm 12.1$ & $56.7 \pm 13.2$ & $55.3 \pm 12.7$ \\
\hline Gender, female & $5,836(76.3)$ & $2,182(76.2)$ & $1,729(75.5)$ & $708(77.6)$ & $387(77.1)$ & $424(75.3)$ & 406 (78.2) \\
\hline Disease duration before b/tsDMARDs & $2.9 \pm 2.8$ & $2.5 \pm 2.4$ & $2.8 \pm 2.6$ & $3.4 \pm 3.3$ & $3.1 \pm 3.0$ & $3.5 \pm 3.2$ & $3.8 \pm 3.6$ \\
\hline Follow-up duration after b/tsDMARDs & $2.9 \pm 2.6$ & $3.9 \pm 2.9$ & $3.0 \pm 2.5$ & $1.9 \pm 1.5$ & $1.6 \pm 1.2$ & $2.0 \pm 1.4$ & $1.1 \pm 0.8$ \\
\hline \multicolumn{8}{|l|}{ Urbanisation status } \\
\hline Urban & $5,741(75.1)$ & 2,088 (73.0) & $1,710(74.7)$ & 698 (76.5) & 394 (78.5) & 445 (79.0) & 406 (78.2) \\
\hline Rural & $1,906(24.9)$ & $774(27.0)$ & 579 (25.3) & $214(23.5)$ & $108(21.5)$ & $118(21.0)$ & $113(21.8)$ \\
\hline \multicolumn{8}{|l|}{ Insured amount, New Taiwan dollars } \\
\hline$<19,200$ & $1,978(25.9)$ & $800(28.0)$ & $584(25.5)$ & $205(22.5)$ & $106(21.1)$ & $149(26.5)$ & $134(25.8)$ \\
\hline $19,200-22,800$ & 2,525 (33.0) & $952(33.3)$ & $767(33.5)$ & $296(32.5)$ & $157(31.3)$ & $193(34.3)$ & $160(30.8)$ \\
\hline$>22,800$ & $3,144(41.1)$ & $1,110(38.8)$ & $938(41.0)$ & $411(45.1)$ & $239(14.6)$ & 221(39.3) & $225(43.4)$ \\
\hline Hospitalised infection within 5 years & $75(1.0)$ & $36(1.3)$ & $20(0.9)$ & $7(0.8)$ & $3(0.6)$ & $5(0.9)$ & $4(0.8)$ \\
\hline \multicolumn{8}{|l|}{ Comorbidities } \\
\hline Hypertension & $1,819(23.8)$ & $679(23.7)$ & $517(22.6)$ & $235(25.8)$ & $108(21.5)$ & $150(26.6)$ & $130(25.1)$ \\
\hline Diabetes mellitus & $736(9.6)$ & $260(9.1)$ & $213(9.3)$ & $87(9.5)$ & $61(12.2)$ & $63(11.2)$ & $52(10.0)$ \\
\hline Pulmonary disease & $539(7.1)$ & $203(7.1)$ & $152(6.6)$ & $50(5.5)$ & $41(8.2)$ & $63(11.2)$ & $30(5.8)$ \\
\hline Chronic kidney disease & $158(2.1)$ & $51(1.8)$ & $28(1.2)$ & $21(2.3)$ & $18(3.6)$ & $26(4.6)$ & $14(2.7)$ \\
\hline Chronic liver disease & $405(5.3)$ & $162(5.7)$ & $119(5.2)$ & $52(5.7)$ & $20(4.0)$ & $31(5.5)$ & $21(4.1)$ \\
\hline Viral hepatitis & $339(4.4)$ & $143(5.0)$ & $96(4.2)$ & $26(2.9)$ & $22(4.4)$ & $35(6.2)$ & $17(3.3)$ \\
\hline \multicolumn{8}{|l|}{ Medications before b/tsDMARDs } \\
\hline Methotrexate (cumulative dose/week, 2.5 mg) & $4.1 \pm 2.0$ & $4.2 \pm 2.0$ & $4.1 \pm 2.1$ & $4.0 \pm 1.9$ & $3.8 \pm 2.1$ & $3.8 \pm 2.0$ & $4.1 \pm 1.9$ \\
\hline Sulfasalazine (cumulative dose/day, 500 mg) & $1.8 \pm 1.6$ & $2.0 \pm 1.6$ & $1.9 \pm 1.6$ & $1.7 \pm 1.6$ & $1.5 \pm 1.6$ & $1.6 \pm 1.6$ & $1.6 \pm 1.6$ \\
\hline Leflunomide (cumulative dose/day, 50 mg) & $0.07 \pm 0.13$ & $0.06 \pm 0.12$ & $0.09 \pm 0.14$ & $0.07 \pm 0.12$ & $0.06 \pm 0.12$ & $0.07 \pm 0.13$ & $0.05 \pm 0.11$ \\
\hline Hydroxychloroquine (cumulative dose/day, 200 mg) & $1.2 \pm 0.8$ & $1.2 \pm 0.8$ & $1.1 \pm 0.8$ & $1.1 \pm 0.8$ & $1.2 \pm 0.8$ & $1.2 \pm 0.7$ & $1.2 \pm 0.7$ \\
\hline Cyclosporin/azathioprin (cumulative DDD/day) & $0.04 \pm 0.13$ & $0.05 \pm 0.13$ & $0.05 \pm 0.14$ & $0.03 \pm 0.12$ & $0.04 \pm 0.12$ & $0.04 \pm 0.13$ & $0.02 \pm 0.09$ \\
\hline Prednisolone equivalent (mg/day) & $6.2 \pm 6.5$ & $6.8 \pm 7.2$ & $6.2 \pm 6.3$ & $5.8 \pm 5.7$ & $6.0 \pm 7.3$ & $5.9 \pm 5.1$ & $4.5 \pm 4.0$ \\
\hline \multicolumn{8}{|l|}{ Concomitant medications with b/tsDMARDs } \\
\hline Methotrexate (mg/week) & $3.8 \pm 4.5$ & $3.5 \pm 2.2$ & $3.7 \pm 3.6$ & $4.13 \pm 4.99$ & $3.7 \pm 6.7$ & $3.52 \pm 2.73$ & $5.3 \pm 10.6$ \\
\hline Sulfasalazine (mg/day) & $119.1 \pm 2,492.8$ & $1.1 \pm 1.5$ & $150.6 \pm 2,787.3$ & $124.0 \pm 2,621.0$ & $335.6 \pm 4,320.4$ & $150.3 \pm 2,636.8$ & $378.6 \pm 4,419.5$ \\
\hline Leflunomide (mg/day) & $0.4 \pm 13.4$ & $0.04 \pm 0.1$ & $0.3 \pm 8.6$ & $0.7 \pm 18.5$ & $1.4 \pm 22.5$ & $0.1 \pm 0.2$ & $2.2 \pm 34.7$ \\
\hline Hydroxychloroquine (mg/day) & $33.4 \pm 590.4$ & $0.8 \pm 0.8$ & $32.6 \pm 584.5$ & $37.7 \pm 641.6$ & $140.3 \pm 1198.2$ & $30.9 \pm 527.3$ & $108.8 \pm 1095.0$ \\
\hline Cyclosporin/azathioprin (mg/day) & $0.03 \pm 0.43$ & $0.02 \pm 0.08$ & $0.05 \pm 0.68$ & $0.02 \pm 0.12$ & $0.02 \pm 0.10$ & $0.02 \pm 0.11$ & $0.04 \pm 0.74$ \\
\hline Prednisolone equivalent (mg/day) & $5.1 \pm 16.1$ & $4.1 \pm 5.1$ & $5.2 \pm 14.3$ & $5.1 \pm 14.3$ & $7.8 \pm 37.0$ & $4.5 \pm 5.3$ & $7.4 \pm 32.0$ \\
\hline
\end{tabular}

Data are presented as mean \pm standard deviation and N (\%).RA, rheumatoid arthritis; b/tsDMARDs: biologics and targeted synthetic disease-modifying antirheumatic drugs; DDD, defined daily dose.

and incidence rate ratios (IRRs) were analysed. The KaplanMeier method was used to compare the cumulative incidence of hospitalised infection among patients categorised by age, urbanisation status, and insured amount. We quantified the associations between covariates and the risk of hospitalised infection by estimating hazard ratios (HRs) with 95\% confidence intervals (CIs) using Cox proportional regression analysis after adjusting for potential confounders. We considered a two-tailed $p<0.05$ as statistically significant. We performed all statistical analyses by SAS statistical software, version 9.3 (SAS Institute, Inc., Cary, NC, USA).

\section{RESULTS}

\section{Characteristics of the Study Population}

A total of 7,647 patients with RA and who received their first b/tsDMARDs between 2003 and 2017 were eligible for analyses. Of them, 6,063 patients received TNFi treatment 
TABLE 2 | Incidence rate of infection requiring hospitalisation in patients receiving b/tsDMARDs.

\begin{tabular}{|c|c|c|c|c|c|c|}
\hline Variable & Total & Event (\%) & Total person-years & Incidence rate $\left(/ 10^{5}\right.$ years) & IRR (95\% CI) & $\begin{array}{c}\text { Log-ran } \\
P\end{array}$ \\
\hline Age at initiating b/tsDMARDs, years & & & & & & $<0.001$ \\
\hline $18-45$ & 1,735 & $3(0.17)$ & 5,677 & 53 & 1 & \\
\hline $45-65$ & 4,358 & $37(0.85)$ & 12,969 & 285 & $5.40(1.66-17.51)$ & \\
\hline$>65$ & 1,554 & $30(1.93)$ & 3,620 & 829 & $15.68(4.79-51.39)$ & \\
\hline Gender & & & & & & 0.138 \\
\hline Female & 5,836 & $49(0.84)$ & 17,221 & 285 & 1 & \\
\hline Male & 1,811 & $21(1.16)$ & 5,046 & 416 & $1.46(0.88-2.44)$ & \\
\hline Urban & & & & & & $<0.001$ \\
\hline Urban & 5,741 & $38(0.66)$ & 16,662 & 228 & 1 & \\
\hline Rural & 1,906 & $32(1.68)$ & 5,605 & 571 & $2.50(1.56-4.01)$ & \\
\hline Insured amount, New Taiwan dollars & & 0.017 & & & & \\
\hline$<19,200$ & 1,978 & $25(1.26)$ & 5,979 & 418 & 1 & \\
\hline $19,200-22,800$ & 2,525 & $29(1.15)$ & 7,024 & 413 & $0.99(0.58-1.69)$ & \\
\hline$>22,800$ & 3,144 & $16(0.51)$ & 9,264 & 173 & $0.41(0.18-0.94)$ & \\
\hline b/tsDMARDs & & & & & & 0.598 \\
\hline Abatacept & 563 & $3(0.53)$ & 1,151 & 261 & 1 & \\
\hline Etanercept & 2,862 & $34(1.19)$ & 11,156 & 305 & $1.17(0.36-3.81)$ & \\
\hline Adalimumab & 2,289 & $26(1.14)$ & 6,875 & 378 & $1.45(0.44-4.79)$ & \\
\hline Golimumab & 912 & $4(0.44)$ & 1,747 & 229 & $0.88(0.20-3.92)$ & \\
\hline Tocilizumab & 502 & $3(0.60)$ & 787 & 381 & $1.46(0.30-7.25)$ & \\
\hline Tofacitinib & 519 & $0(0.00)$ & 551 & 0 & NA & \\
\hline
\end{tabular}

$R A$, rheumatoid arthritis; b/tsDMARDs: biologics and targeted synthetic disease-modifying antirheumatic drugs.

(etanercept $n=2,862$; adalimumab $n=2,289$; golimumab $n$ $=912$ ), 1,065 received non-TNFi bDMARDs (tocilizumab $n=$ 502; abatacept $n=563$ ), and 519 patients were treated with tsDMARD (tofacitinib).

The mean age was $53.9 \pm 12.8$ years; $76.3 \%$ of them were female. The duration between diagnosis of RA and initiation of b/tsDMARDs was $2.9 \pm 2.8$ years, while the duration from initiation of b/tsDMARDs to hospitalised infection was $2.9 \pm$ 2.6 years. A distinct socioeconomic status was found among patients with RA and received b/tsDMARDs, with $75.1 \%$ of them living in an urban area, and $41.4 \%$ of them having had a relatively high income. Notably, $\sim 1 \%$ of the enrolled patients had hospitalised infection within 5 years. Table 1 further summarises comorbidities, medications prior to the initiation of b/tsDMARDs, and concomitant medications with the use of b/tsDMARDs (Table 1).

\section{Incidence Rate and IRRs of Hospitalised Infection}

Table 2 shows the incidence rates of hospitalised infection and the IRRs with $95 \%$ CIs. We found that age affected the incidence of hospitalised infection, and the incidence rates (per 100,000 person-years) were 829 ( $\geq 65$ group), 285 (46-65 group), and 53 (18-45 group), respectively. The IRR was 15.68 (4.79-51.39) in those older than 65 years and $5.40(1.66-17.51)$ in patients whose ages ranged from 45 and 65 years compared with the incidence rate among patients whose ages are between 18 and 45 years. Socioeconomic status also had a crucial impact on incident hospitalised infection. The IRR was 2.50 (1.56-4.01) in patients living in a rural area compared to those living in an urban area, whereas the IRRs were $0.41(0.18-0.94)$ in patients with the highest income category compared with patients with the lowest income category. With regard to the effect of distinct $\mathrm{b} / \mathrm{tsDMARDs}$ on incident hospitalised infection, we found no significant difference in incident hospitalised infection among the distinct b/tsDMARDs (Table 2).

\section{Risk Factors for Hospitalised Infection Among RA Patients Receiving b/tsDMARDs}

We then used the log-rank analyses to demonstrate the impact of age, urbanisation status, and income on the incident hospitalised infection (Figure 1). We next estimated the risk for hospitalised infection using univariate and multivariable Cox proportional regression analyses (Table 3 ). We found that age independently affected the risk for hospitalised infection at a dose-response manner ( $\geq 65$ age group, HR 6.44, 95\% CI 1.82-22.80; 4564 age group, HR 3.49, 95\% CI 1.04-11.70). A high income was inversely associated with the incident hospitalised infection (HR 0.48, 95\% CI 0.23-0.96). Living in a rural area tended to be a risk factor for hospitalised infection, although not reaching a statistical significance (HR 1.76, 95\% CI 0.983.14). We also found that hospitalisations within 5 years (HR 5.63, 95\% CI 1.91-16.62) were a robust predictor for incident hospitalised infection after the initiation of b/tsDMARDs in 
patients with RA. With regard to the effects of previously used and concomitant medication on the hospitalised infection, we noted that the usage of sulfasalazine was somehow inversely associated with hospitalised infection (HR 0.71, 95\% CI 0.560.91 ), and the concomitant corticosteroid (HR 1.07, 95\% CI 1.051.09 prednisolone equivalent per $1 \mathrm{mg} /$ day increment), as well as leflunomide [HR 18.57, 95\% CI 1.52-227.06, cumulative dose $(50 \mathrm{mg})$ per day increment], was associated with the incident hospitalised infection. We also analysed data in patients receiving csDMARDs alone. Consistently, the data of 21,361 subjects with RA receiving csDMARDs alone showed that incident hospitalised infection was associated with hospitalisation for infection within 5 years (aHR 2.43, 95\% CI 1.35-4.36), whereas a high-income category had an inverse association (aHR 0.43, 95\% CI 0.31-0.60) (Supplementary Tables 2-4).

\section{DISCUSSION}

To the best of our knowledge, this is the first populationbased study to explore the impact of socioeconomic status on the risk for hospitalised infection among patients with RA receiving $\mathrm{b} / \mathrm{tsDMARDs}$. We found that age was associated with hospitalised infection in a dose-response manner and prior hospitalised infection was a strong predictor for hospitalised infection after the initiation of b/tsDMARDs in patients with RA. Notably, we found that high socioeconomic status, particularly a high income, was inversely associated with incident hospitalised infection. Additionally, we noted that concomitant use of corticosteroid was also associated with the development of hospitalised infection among patients with RA receiving b/tsDMARDs.

Surprisingly, socioeconomic status has been a proven determinant for incident sepsis in the general population (12, 17); however, the role of socioeconomic status in incident infection among patients with RA receiving b/tsDMARDs is largely unknown although a number of studies have shown that socioeconomic status has a crucial impact on the initiation of $\mathrm{b} /$ tsDMARDs $(18,19)$. Molina et al. investigating 1,209 patients with RA in San Antonio, found that a lower socioeconomic status, defined by education level, household income, and occupation, was associated with a delay of first cDMARDs (8.5 \pm 10.2 vs. $6.1 \pm 7.9$ years; $p=0.002$ ) (18). One Swedish study, conducted by Frisell et al. addressed factors associated with the first bDMARD among 9,310 patients with RA and found that patients receiving non-TNFi as the first bDMARD had lower socioeconomic status and were more likely to have recent serious infections than those receiving TNFi (19). The finding of Frisell et al. implicates the potential concern of the development of infection in administering TNFi as the first bDMARD in RA patients with low socioeconomic status. Our recently published study, investigating risk for sepsis in patients receiving TNFi for immune-mediated inflammatory diseases, including RA, ankylosing spondylitis, psoriasis, psoriatic arthritis, Crohn's disease, and ulcerative colitis, identified that risk for sepsis was associated with lower levels of urbanisation and payrollrelated insured amount (10). In the present study, we further

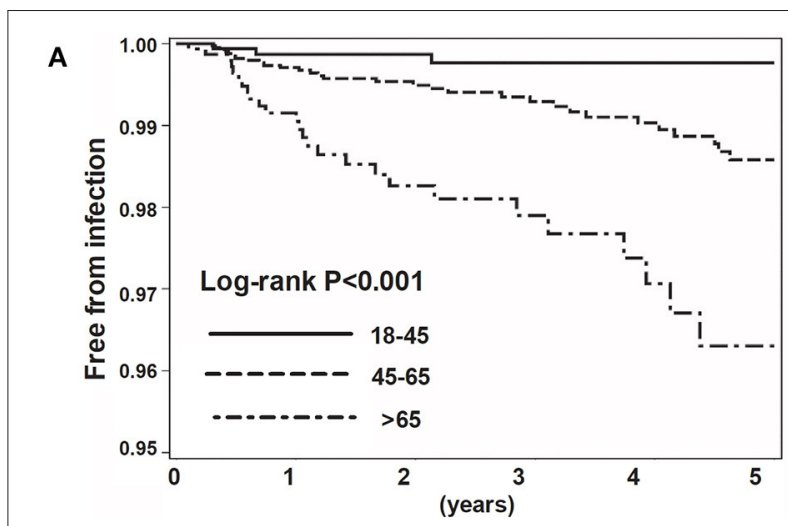

B

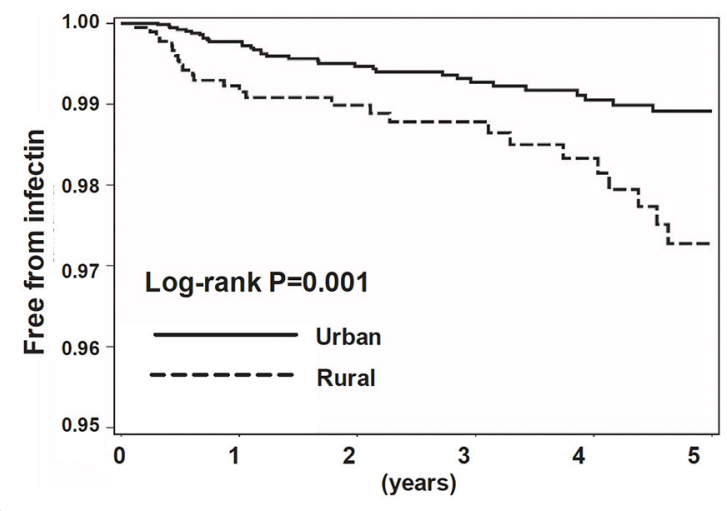

C

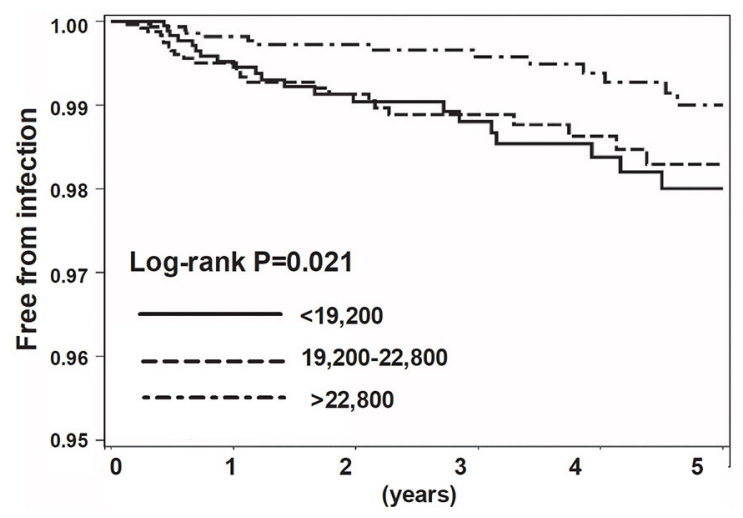

FIGURE 1 | Kaplan-Meier survival curve for incidental hospitalised infection. Categorised by age (A), urbanisation status (B), and insured amount (C).

demonstrated the robust association between hospitalised infection and insured amount in RA patients receiving distinct bDMARDs or tsDMARDs, and we think these findings indicate that socioeconomic status must be considered in infection risk stratification in patients with RA receiving b/tsDMARDs.

Age plays a substantial role in sepsis; however, the impact of age on incident infection in patients with RA appears to vary with studies due to the distinct target to treat strategy, use of b/tsDMARDs, and concomitant GCs (20-22). Increasing evidence has shown that the concern regarding the safety issue of b/tsDMARDs in elderly patients with RA might somehow deprive those patients of optimal disease control and quality of 
TABLE 3 | Crude and adjusted hazard ratios for the association between incident hospitalised infection and variables.

\begin{tabular}{|c|c|c|c|c|}
\hline & HR (95\% Cl) & $p$ value & aHR $(95 \% \mathrm{Cl})$ & $p$ value \\
\hline \multicolumn{5}{|l|}{ Age at initiating b/tsDMARDs, years } \\
\hline $45-65$ & $4.24(1.29-13.88)$ & 0.017 & $3.49(1.04-11.70)$ & 0.043 \\
\hline$>65$ & $12.33(3.73-40.77)$ & $<0.001$ & $6.44(1.82-22.80)$ & 0.004 \\
\hline Gender-male & $1.25(0.70-2.21)$ & 0.454 & $0.96(0.52-1.75)$ & 0.887 \\
\hline Urban & Ref. & & Ref. & \\
\hline Rural & $2.35(1.41-3.93)$ & 0.001 & $1.76(0.98-3.14)$ & 0.058 \\
\hline \multicolumn{5}{|l|}{ Insured amount, New Taiwan dollars } \\
\hline$<19,200$ & Ref. & & Ref. & \\
\hline $19,200-22,800$ & $0.95(0.53-1.71)$ & 0.864 & $0.68(0.36-1.27)$ & 0.226 \\
\hline Hypertension & $2.57(1.53-4.31)$ & 0.000 & $1.39(0.78-2.48)$ & 0.259 \\
\hline Diabetes mellitus & $3.10(1.70-5.65)$ & 0.000 & $1.83(0.97-3.45)$ & 0.062 \\
\hline Pulmonary disease & $2.69(1.32-5.47)$ & 0.006 & $1.31(0.61-2.83)$ & 0.493 \\
\hline Chronic kidney disease & $4.31(1.56-11.90)$ & 0.005 & $1.91(0.63-5.77)$ & 0.251 \\
\hline Chronic liver disease & $1.81(0.72-4.52)$ & 0.205 & $1.08(0.39-2.98)$ & 0.887 \\
\hline Viral hepatitis & $3.85(1.82-8.10)$ & 0.000 & $2.90(1.20-6.99)$ & 0.018 \\
\hline \multicolumn{5}{|l|}{ b/tsDMARDs } \\
\hline Abatacept & Ref. & & Ref. & \\
\hline Etanercept & $1.12(0.34-3.73)$ & 0.854 & $1.52(0.45-5.14)$ & 0.500 \\
\hline Adalimumab & $1.59(0.48-5.31)$ & 0.448 & $2.15(0.63-7.26)$ & 0.220 \\
\hline Sulfasalazine (cumulative dose/day, 500 mg) & $0.84(0.71-0.98)$ & 0.031 & $0.71(0.56-0.91)$ & 0.007 \\
\hline Leflunomide (cumulative dose/day, 50 mg) & $17.04(3.43-84.72)$ & 0.001 & $1.33(0.11-16.48)$ & 0.825 \\
\hline Hydroxychloroquine (cumulative dose/day, 200 mg) & $1.00(0.73-1.39)$ & 0.979 & $1.06(0.69-1.63)$ & 0.782 \\
\hline Cyclosporin/azathioprin (cumulative DDD/day) & $3.38(0.76-15.10)$ & 0.110 & $1.21(0.14-10.14)$ & 0.863 \\
\hline \multicolumn{5}{|l|}{ Concomitant medications with b/tsDMARDs } \\
\hline Prednisolone equivalent, mg/day & $1.06(1.05-1.08)$ & $<0.001$ & $1.07(1.05-1.09)$ & $<0.001$ \\
\hline Methotrexate (cumulative dose/week, 2.5 mg) & $0.84(0.74-0.95)$ & 0.007 & $1.11(0.94-1.32)$ & 0.221 \\
\hline Sulfasalazine (cumulative dose/day, 500 mg) & $1.08(0.90-1.30)$ & 0.394 & $1.29(0.99-1.67)$ & 0.059 \\
\hline Leflunomide (cumulative dose/day, 50 mg) & $70.18(13.72-358.98)$ & $<0.001$ & $18.57(1.52-227.06)$ & 0.022 \\
\hline Hydroxychloroquine (cumulative dose/day, 200 mg) & $1.21(0.88-1.67)$ & 0.239 & $0.99(0.66-1.47)$ & 0.950 \\
\hline Cyclosporin/azathioprin (cumulative DDD/day) & $9.96(1.92-51.61)$ & 0.006 & $2.75(0.21-36.87)$ & 0.446 \\
\hline
\end{tabular}

RA, rheumatoid arthritis; b/tsDMARDs, biologics and targeted synthetic disease-modifying antirheumatic drugs.

life (20). Tatangelo et al. conducting a population-based study in Canada to explore factors for the duration from the first csDMARDs to the first bDMARDs in patients with RA, reported a positive association between increasing age and longer time to receipt of a bDMARD (21). Furthermore, Black et al. investigated the use of GCs among patients with RA in the United Kingdom primary care research database and found that nearly half of patients with incident RA received GCs and GCs were more 
frequently prescribed as elderly patients with RA (22). Given that the efficacy of b/tsDMARDs appears unrelated to age, the increased use of GC, instead of b/tsDMARDs, in elderly patients with RA may possibly lead to an increased risk for infection (23). In line with our data, Widdifield et al. conducted a nested casecontrol study using administrative data across 1992-2010 with 86,039 patients with RA in Ontario, demonstrating that age was an independent predictor for infection requiring hospitalisation or emergency department visit (aOR, 1.05; 95\% CI 1.04-1.06) (24). In the present study, we found that the association between age ( $>65$ years) and incident hospitalised infection appears to be higher in RA patients receiving b/tsDMARD (aHR 6.44, 95\% CI 1.82-22.80) than those receiving csDMARDs (aHR 3.18, 95\% CI 2.19-4.60). This evidence including our data suggests a crucial need for vigilance of infection in elderly patients receiving $\mathrm{b} / \mathrm{tsDMARDs}$.

A number of studies have shown a high proportion of patients who were readmitted after discharge from the hospital for the infectious disease $(10,25,26)$. Prescott et al. using Medicare claims with 2,617 severe sepsis survivors in the US, found that up to $42.7 \%$ severe sepsis survivors were readmitted within 3 months, mainly due to sepsis and pneumonia (25). One Taiwanese population-based study also reported that septic patients had a higher risk of further sepsis than those in ageand sex-matched non-septic controls (35.0 vs. 4.3\%) (26). Our previous study found that recent sepsis within 3 months before TNFi initiation was a robust predictor for incident sepsis in patients receiving TNFi in patients with IMIDs (10). In the present study, we further found a robust impact of a history of hospitalised infection on the development of hospitalised infection after receiving b/tsDMARDs among patients with RA. As shown in the aforementioned Swedish study, the physician tends to prescribe non-TNFi, instead of TNFi, as the first bDMARD in RA patients with recent serious infection, indicating the concern of physicians with regard to further infection in prescribing TNFi (19). Therefore, we think that there is a need for vigilance in the follow-up of RA patients who had a history of serious infection and have received b/tsDMARDs; however, more studies are warranted for the optimal strategy in selecting $\mathrm{b} / \mathrm{tsDMARD}$ in RA patients with a history of infection, particularly those with a low socioeconomic status.

Intriguingly, we found that the concomitant use of leflunomide was highly associated with the incident hospitalised infection. Given that leflunomide is indicated as the second-line csDMARD in Taiwan after the ineffective/intolerable first-line csDMARD therapy, including methotrexate, sulfasalazine, or cyclosporine, we thus postulate that the high disease activity may potentially underlie the strong association between use of leflunomide and hospitalised infection.

The strength of this study is the use of a nationwide population-based cohort, containing comprehensive data regarding socioeconomic status, to mitigate selection bias. However, some limitations remain to be acknowledged. First is the concern for the accuracy of the diagnosis in claims data; however, the diagnosis of RA was validated by at least two qualified rheumatologists after checking the original medical data. Second, the potential socioeconomic status-associated confounding factors, including the use of tobacco and alcohol, were unavailable in the NHIRD. However, the majority of enrolled patients with RA in the present study were females (76.3\%), and the prevalence of tobacco use among females in Taiwan was marked low (2.6\%) (27). Third, the study results may not be applied to non-Taiwanese populations with distinct overall socioeconomic status. Fourth, a number of biologics, including anakinra, infliximab, certolizumab, and rituximab (proven as the second-line bDMARD in Taiwan), were not studied given that these biologics were not approved as first-line biologics for RA during the study period in Taiwan. Another limitation is the lack of controls, including disease severity-matched patients with RA but who did not undergo b/tsDMARD therapy and patients receiving b/tsDMARDs for diseases other than RA. However, we have shown consistent findings among patients with RA receiving csDMARDs alone (Supplementary Tables 2-4).

\section{CONCLUSION}

This nationwide, population-based, cohort study addressed risk factors for hospitalised infection in patients with RA receiving $\mathrm{b} / \mathrm{tsDMARDs}$. In line with other studies including our previous study, we found that age and prior hospitalised infection were the robust predictors for hospitalised infection after the initiation of b/tsDMARDs in patients with RA. Notably, we found that high socioeconomic status, including a high income and living in an urbanised area, appeared to be inversely associated with incident hospitalised infection. Moreover, concomitant use of corticosteroid also associated with an increased risk for hospitalised infection. These findings, particularly the identification of the impact of socioeconomic status, should be crucial for risk stratification in patients with RA receiving b/tsDMARDs.

\section{DATA AVAILABILITY STATEMENT}

The original contributions presented in the study are included in the article/Supplementary Material, further inquiries can be directed to the corresponding authors.

\section{ETHICS STATEMENT}

The studies involving human participants were reviewed and approved by IRB of Taichung Veterans General Hospital (IRB TCVGH number: CE19038A). The ethics committee waived the requirement of written informed consent for participation.

\section{AUTHOR CONTRIBUTIONS}

$\mathrm{H}-\mathrm{HC}, \mathrm{C}-\mathrm{YW}$, and W-CC: conceived, designed the experiments, and wrote the paper. $\mathrm{H}-\mathrm{HC}$ and $\mathrm{C}-\mathrm{HL}$ : acquired the data. C-HL and W-CC: contributed the materials and analysis tools. All authors contributed to the article and approved the submitted version. 


\section{FUNDING}

This study was supported in part by grants from Taichung Veterans General Hospital, Taiwan (TCVGH-1097321D, TCVGH-1094405D, and TCVGH-1094406D). The funders had no role in the study design, data collection and analysis, decision to publish, or preparation of the manuscript.

\section{ACKNOWLEDGMENTS}

This study is based on data from the NHIRD in Taiwan, and the interpretation of data does not

\section{REFERENCES}

1. Scott DL, Wolfe F, Huizinga TW. Rheumatoid arthritis. Lancet. (2010) 376:1094-8. doi: 10.1016/S0140-6736(10)60826-4

2. Cross M, Smith E, Hoy D, Carmona L, Wolfe F, Vos T, et al. The global burden of rheumatoid arthritis: estimates from the global burden of disease 2010 study. Ann Rheum Dis. (2014) 73:1316-22. doi: 10.1136/annrheumdis-2013-204627

3. Fazal SA, Khan M, Nishi SE, Alam F, Zarin N, Bari MT, et al. A clinical update and global economic burden of rheumatoid arthritis. Endocr Metab Immune Disord Drug Targets. (2018) 18:98-109. doi: 10.2174/1871530317666171114122417

4. Huscher D, Mittendorf T, von Hinuber U, Kotter I, Hoese G, Pfafflin A, et al. Evolution of cost structures in rheumatoid arthritis over the past decade. Ann Rheum Dis. (2015) 74:738-45. doi: 10.1136/annrheumdis-2013-2 04311

5. Taylor PC. Clinical efficacy of launched JAK inhibitors in rheumatoid arthritis. Rheumatology. (2019) 58:i17-26. doi: 10.1093/rheumatology/key225

6. Rutherford AI, Subesinghe S, Hyrich KL, Galloway JB. Serious infection across biologic-treated patients with rheumatoid arthritis: results from the British Society for Rheumatology Biologics Register for Rheumatoid Arthritis. Ann Rheum Dis. (2018) 77:905-910. doi: 10.1136/annrheumdis-2017-2 12825

7. Machado MA, Moura CS, Guerra SF, Curtis JR, Abrahamowicz M, Bernatsky S. Effectiveness and safety of tofacitinib in rheumatoid arthritis: a cohort study. Arthritis Res Ther. (2018) 20:60. doi: 10.1186/s13075-0181539-6

8. Simon TA, Boers M, Hochberg M, Baker N, Skovron ML, Ray N, et al. Comparative risk of malignancies and infections in patients with rheumatoid arthritis initiating abatacept versus other biologics: a multi-database real-world study. Arthritis Res Ther. (2019) 21:228. doi: 10.1186/s13075-019-1992-x

9. Ozen G, Pedro S, England BR, Mehta B, Wolfe F, Michaud K. Risk of serious infection in patients with rheumatoid arthritis treated with biologic versus nonbiologic disease-modifying antirheumatic drugs. ACR Open Rheumatol. (2019) 1:424-32. doi: 10.1002/acr2.11064

10. Chao WC, Wang CY, Hsu BC, Lin $\mathrm{CH}$, Huang WN, Chen $\mathrm{YH}$, et al. Factors associated with sepsis risk in immunemediated inflammatory diseases receiving tumor necrosis factor inhibitors: a nationwide study. Ther Adv Musculoskelet Dis. (2020) 12:1759720X20929208. doi: 10.1177/1759720X20929208

11. Gron KL, Arkema EV, Glintborg B, Mehnert F, Ostergaard M, Dreyer $\mathrm{L}$, et al. Risk of serious infections in patients with rheumatoid arthritis treated in routine care with abatacept, rituximab and tocilizumab in Denmark and Sweden. Ann Rheum Dis. (2019) 78:320-7. doi: 10.1136/annrheumdis-2018-214326

12. Baker MG, Barnard LT, Kvalsvig A, Verrall A, Zhang J, Keall M, et al. Increasing incidence of serious infectious diseases and inequalities in New Zealand: a national epidemiological study. Lancet. (2012) 379:11129. doi: 10.1016/S0140-6736(11)61780-7 represent those of the NHIRD. The authors thank the statistical work by the Biostatistics Task Force and Healthcare Service Research Center of Taichung Veterans General Hospital.

\section{SUPPLEMENTARY MATERIAL}

The Supplementary Material for this article can be found online at: https://www.frontiersin.org/articles/10.3389/fmed. 2021.696167/full\#supplementary-material
13. Rush B, Wiskar K, Celi LA, Walley KR, Russell JA, McDermid RC, et al. Association of household income level and in-hospital mortality in patients with sepsis: a nationwide retrospective cohort analysis. J Intensive Care Med. (2018) 33:551-6. doi: 10.1177/0885066617703338

14. Doran MF, Crowson CS, Pond GR, O'Fallon WM, Gabriel SE. Frequency of infection in patients with rheumatoid arthritis compared with controls: a population-based study. Arthritis Rheum. (2002) 46:2287-93. doi: 10.1002/art.10524

15. Cheng TM. Taiwan's new national health insurance program: genesis and experience so far. Health Affairs. (2003) 22:61-76. doi: 10.1377/hlthaff.22.3.61

16. Lin $\mathrm{CH}$, Wang JL, Chen HH, Hsu JY, Chao WC. Shared prenatal impacts among childhood asthma, allergic rhinitis and atopic dermatitis: a population-based study. Allergy Asthma Clin Immunol. (2019) 15:52. doi: 10.1186/s13223-019-0365-y

17. Donnelly JP, Lakkur S, Judd SE, Levitan EB, Griffin R, Howard G, et al. Association of neighborhood socioeconomic status with risk of infection and sepsis. Clin Infect Dis. (2018) 66:1940-7. doi: 10.1093/cid/ci x1109

18. Molina E, Del Rincon I, Restrepo JF, Battafarano DF, Escalante A. Association of socioeconomic status with treatment delays, disease activity, joint damage, and disability in rheumatoid arthritis. Arthritis Care Res. (2015) 67:9406. doi: 10.1002/acr.22542

19. Frisell T, Baecklund E, Bengtsson K, Di Giuseppe D, Forsblad-d'Elia $\mathrm{H}$, Askling $\mathrm{J}$, et al. Patient characteristics influence the choice of biological drug in RA, and will make non-TNFi biologics appear more harmful than TNFi biologics. Ann Rheum Dis. (2018) 77:6507. doi: 10.1136/annrheumdis-2017-212395

20. Oishi S, Wendling D, Sibilia J, Job-Deslandre C, Guillevin L, Benichou J, et al. Treatment of active rheumatoid arthritis: comparison of patients younger vs older than 75 years (CORPUS cohort). Hum Vaccin Immunother. (2018) 14:2612-7. doi: 10.1080/21645515.2018.1522470

21. Tatangelo M, Tomlinson G, Paterson JM, Ahluwalia V, Kopp A, Gomes T, et al. Association of patient, prescriber, and region with the initiation of first prescription of biologic disease-modifying antirheumatic drug among older patients with rheumatoid arthritis and identical health insurance coverage. JAMA Netw Open. (2019) 2:e1917053. doi: 10.1001/jamanetworkopen.2019.17053

22. Black RJ, Joseph RM, Brown B, Movahedi M, Lunt M, Dixon WG. Half of U.K. patients with rheumatoid arthritis are prescribed oral glucocorticoid therapy in primary care: a retrospective drug utilisation study. Arthritis Res Ther. (2015) 17:375. doi: 10.1186/s13075-0150895-8

23. Koller MD, Aletaha D, Funovits J, Pangan A, Baker D, Smolen JS. Response of elderly patients with rheumatoid arthritis to methotrexate or TNF inhibitors compared with younger patients. Rheumatology. (2009) 48:157580. doi: 10.1093/rheumatology/kep291

24. Widdifield J, Bernatsky S, Paterson JM, Gunraj N, Thorne JC, Pope $J$, et al. Serious infections in a population-based cohort of 86,039 seniors with rheumatoid arthritis. Arthritis Care Res. (2013) 65:35361. doi: $10.1002 /$ acr.21812 
25. Prescott HC, Langa KM, Iwashyna TJ. Readmission diagnoses after hospitalisation for severe sepsis and other acute medical conditions. JAMA. (2015) 313:1055-7. doi: 10.1001/jama.201 5.1410

26. Shen HN, Lu CL, Yang HH. Risk of recurrence after surviving severe sepsis: a matched cohort study. Crit Care Med. (2016) 44:1833-41. doi: 10.1097/CCM.0000000000001824

27. Taiwan tobacco control annual report 2018. Health Promotion Administration, Ministry of Health and Welfare, Taiwan. (2018). Available online at: https://health99.hpa.gov.tw/media/public/pdf/22077.pdf (accessed October 10, 2019).
Conflict of Interest: The authors declare that the research was conducted in the absence of any commercial or financial relationships that could be construed as a potential conflict of interest.

Copyright (c) 2021 Chen, Lin, Wang and Chao. This is an open-access article distributed under the terms of the Creative Commons Attribution License (CC BY). The use, distribution or reproduction in other forums is permitted, provided the original author(s) and the copyright owner(s) are credited and that the original publication in this journal is cited, in accordance with accepted academic practice. No use, distribution or reproduction is permitted which does not comply with these terms. 